\title{
Study of resistance of ultra-high molecular weight polyethylene to mechanochemical and radiation exposure
}

\author{
Kamila M. Khassenova ${ }^{1-}$, Sergey V. Vosmerikov ${ }^{2}$ \\ ${ }^{1}$ Novosibirsk State Technical University, 630073, Novosibirsk, Russia \\ ${ }^{2}$ Institute of Solid State Chemistry and Mechanochemistry SB RAS, 630128, Novosibirsk, Russia
}

\begin{abstract}
Ultra-high molecular weight polyethylene is a promising composite material to protect against ionizing radiation. The effect of mechanical activation and radiation exposure on the polymer structure has been studied. Mechanical activation of ultra-high molecular weight polyethylene was carried out for $30 \mathrm{~s}, 1$, and $2 \mathrm{~min}$ in a high-energy watercooled planetary ball mill AGO-2, followed by its further investigation using X-ray diffraction, X-ray crystallography, IR spectroscopy, scanning electron microscopy, and differential scanning calorimetry.
\end{abstract}

The problem of individual protection of people, portable electronic devices, and equipment from multifaceted ionizing radiation is still central and currently, an ongoing search for new materials to solve this problem is being conducted. Polymer composites, where individual absorbing abilities of polymers and modifiers are used, are very promising for obtaining lightweight and easily moldable materials. Polymers with a high degree of polymerization are being used for the development of composite materials for radiation shielding, and industrial production availability is an important factor.

In this study commercially available ultra-high molecular weight polyethylene (UHMWPE) was used, which is a promising material for radiation shielding because of its tendency for cross-linking between macromolecules when irradiated, high molecular weight, and high hydrogen content. The molecular weight of the polymer is $4 \cdot 10^{6} \mathrm{~g} / \mathrm{mol}$, the particle size is $100-160 \mu \mathrm{m}$.

UHMWPE has high tenacity, wear resistance, toughness very high resistance to aggressive agents, water, and temperature in the range from -200 to $+90^{\circ} \mathrm{C}$, which results in vast application possibilities [1].

Using a mechanochemical approach for obtaining composite polymer materials means that besides resistance to radiation the polymer component has to show good mechanical qualities.

Since during the formation process of filled polymer composite materials, both polymers and inorganic fillers are undergoing not only mechanical activation (MA) but also

- Corresponding author: kmkhassenova@gmail.com 
the pressure and heating treatment by hot pressing, the impact of these processes on structural-morphological properties of UHMWPE was studied.

UHMWPE is an amorphocrystalline material consisting of crystalline folded polymer structures and amorphous regions, where molecular chains are in a disordered state. Inside crystalline formations, carbon atoms are in planar zigzag conformation. Crystalline structure is described as an orthorhombic phase with unit cell parameters $a=0.74 \mathrm{~nm}$, $b=0.493 \mathrm{~nm}, c=0.254 \mathrm{~nm}$ [2]. UHMWPE crystallites are predominantly (110)-oriented because the reflection intensity of this plane surpasses by far the other ones (Fig 1, curve 1). The size of crystallites is about $20 \mathrm{~nm}$.

The amorphous region of UHMWPE consists of randomly oriented and continuous polymer chains that are a part of two or more nearby crystallites.

Mechanochemical activation was carried out in high-energy planetary ball mill AGO-2 with water cooling in the atmosphere of argon. The volume of the jar is $250 \mathrm{~cm}^{3}$, the ball diameter is $5 \mathrm{~mm}$, the weight of the balls is $200 \mathrm{~g}$, the sample weight is $10 \mathrm{~g}$, the rotation speed of the jars around the common axis is $\sim 1000 \mathrm{rpm}$.

Under shock shear impulse effect implemented in AGO-2, an increase of integral intensity of the reflection (010) is recorded on X-ray diffraction pattern (XRD) of pure UHMWPE (Fig. 1, curves 2-4). The size of UHMWPE crystallites after $2 \mathrm{~min}$ of MA slightly changes from the initial $26 \mathrm{~nm}$ to $29 \mathrm{~nm}$ after MA.

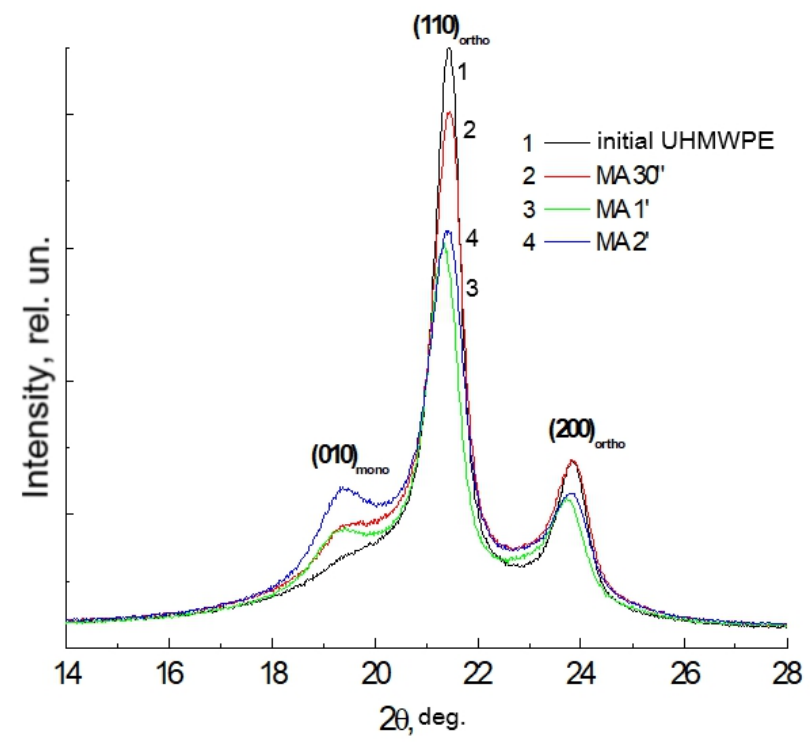

Fig. 1. X-ray diffraction patterns of UHMWPE in an initial state (curve 1) and after mechanical activation (curves 2-4).

On IR spectra of UHMWPE before and after MA the following absorption bands are being registered: a $2913 \mathrm{~cm}^{-1}$ and $2846 \mathrm{~cm}^{-1}$ doublet, pertaining to symmetric and asymmetric stretches $v\left(\mathrm{CH}_{2}\right)$, a $1472 \mathrm{~cm}^{-1}$ and $1463 \mathrm{~cm}^{-1}$ doublet - a bending scissoring vibration of trans methyl group $\delta\left(\mathrm{CH}_{2}\right), 730 \mathrm{~cm}^{-1}$ and $720 \mathrm{~cm}^{-1}$ - rocking vibrations $\rho\left(\mathrm{CH}_{2}\right)$ (Fig. 2). In addition, in IR spectra of polyethylene in the range of bending vibrations 1450$1475 \mathrm{~cm}^{-1}$ absorption bands of methyl groups can be identified that are pertained to its transitional and amorphous regions:

- 1471,1468 , and $1464 \mathrm{~cm}^{-1}$ bands - to mixed $\delta$-vibrations of gauche- and trans-methyl chains in the transitional region; 
- $1466 \mathrm{~cm}^{-1}-$ to $\delta$-vibrations of gauche-methyl groups in the amorphous region, and $1456 \mathrm{~cm}^{-1}$ - to mixed disordered $\delta$-vibrations of gauche- and trans-methyl chains in the amorphous region.

1303,1370 , and $1350 \mathrm{~cm}^{-1}$ bands also pertain to the amorphous region of polyethylene, the latter are conformation sensitive and correspond to trans- (stretched) and gauche(contracted) rotational isomers.

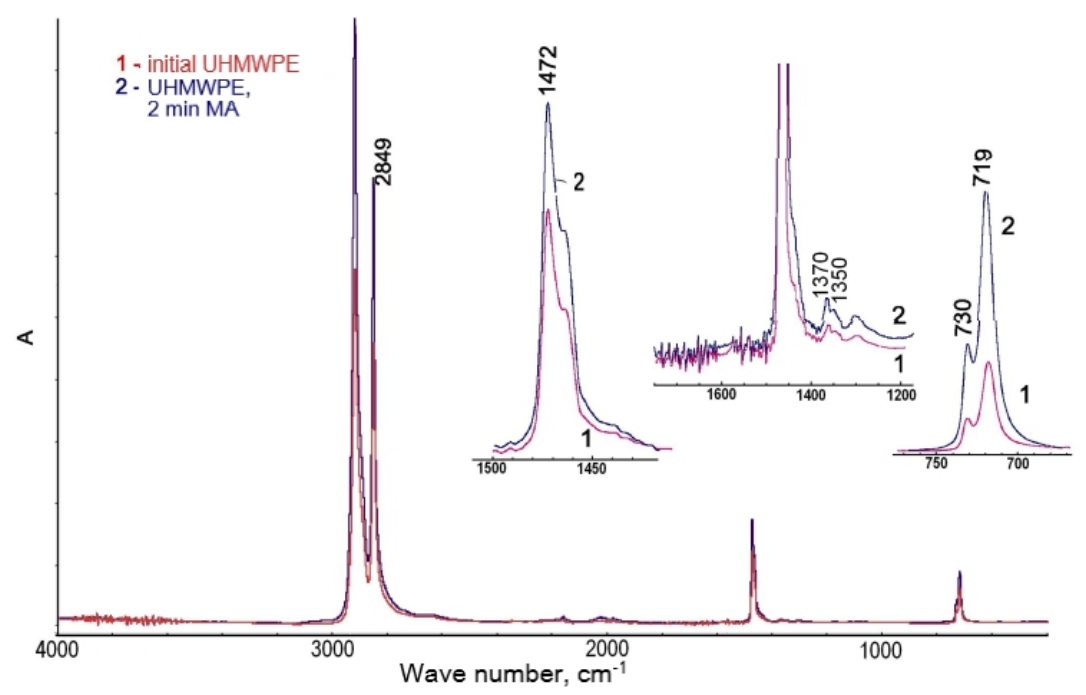

Fig. 2. IR spectra of UHMWPE in an initial state (1) and after mechanical activation (2).

Considering the relation of viscoelastic behavior of the polymer to the speed and time of deformation, it is believed that with a short duration time of high-speed impulse mechanical impact the UHMWPE powder mostly acts as an elastic body. Deformation in globe structure is associated with the segmental mobility of molecules, leading to a decrease in the intermolecular distance with the applied stress field. As a result, the overall increase in the intensity of absorption bands on IR spectra is observed. The amount of energy in the chosen MA conditions is not enough to break the C-C bonds [3], but enough to change the submolecular structure of the polymer partially. The degree of crystallinity of UHMWPE after MA is increased slightly from 40 to 44 vol.\%.

It is well known that creep of polymer is mainly associated with the development of deformation that is driven by conformational transformations. The latter occur largely in macromolecules of amorphous regions because the intermolecular forces between tie macromolecules, that form amorphous regions, are much lower than between macromolecules that form crystalline regions. Namely, an increase of amorphous region with longer time of MA leads to creep development.

Scanning electron microscopy (SEM) images of UHMWPE particles indicate a decrease in particle size up to 2-4 times after MA for 2 min comparing to the initial (Fig. 3). The shape of the particles changes from globular to flake.

Based on differential scanning calorimetry (DSC) data, melting of the initial UHMWPE amorphocrystalline structure begins at $90-95^{\circ} \mathrm{C}$ and stops at $\sim 180-200^{\circ} \mathrm{C}$. A faint peak at around $120^{\circ} \mathrm{C}$ indicates that there is a molecular structure with a lower melting temperature in the polymer.

According to X-ray diffraction patterns, neutron irradiation does not lead to the change in phase composition of the samples, meanwhile conducts structural changes of the components, which affects the mechanical properties of the material. Increasing the 
absorbed dose increases the intensity of reflections of the polymer that is caused by an increase in the size of its crystallites from 26 to $62 \mathrm{~nm}$. In addition, there is an increase in microstress levels caused by appearing irradiation defects. Mechanical activation leads to the growth of UHMWPE crystallites with subsequent milling, which is also followed by microstress growth. Based on X-ray crystallography (XRC), during irradiation UHMWPE crystallites increase 2.3 times, and unit cell volume increases by $4 \%$. Mechanochemical activation promotes the decrease in the growth rate of UHMWPE crystallites 1.2 times and the increase of unit cell volume up to $0.4 \%$. However, the selected modes of MA and irradiation do not cause significant changes in mechanical properties of the polymer.

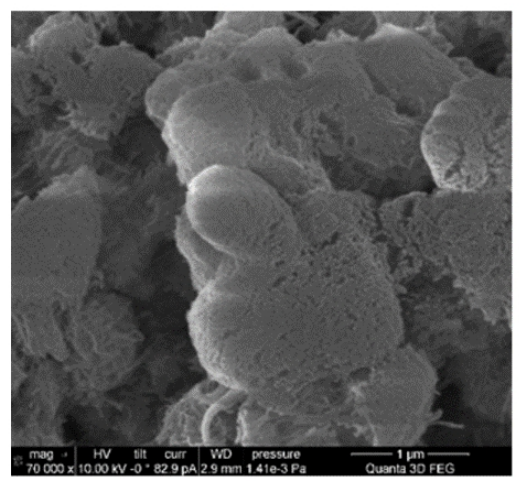

a

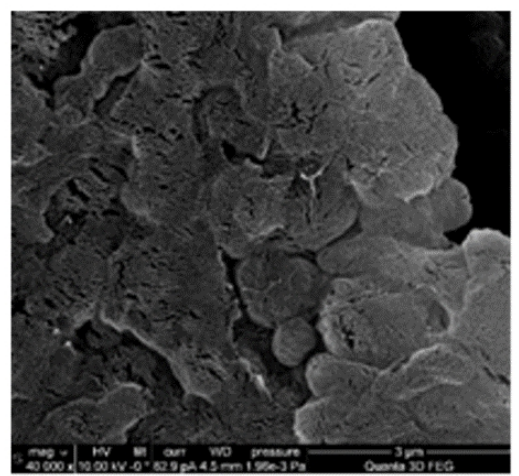

b

Fig. 3. Scanning electron microscopy images of UHMWPE in an initial state (a) and after mechanical activation (b).

Analysis of radiation attenuation $(\lambda=1.512 \AA)$ showed a measured attenuation coefficient for the UHMWPE samples of $0.20 \mathrm{~mm}^{-1}$.

The work was carried out within the state assignment of the Institute of Solid State Chemistry and Mechanochemistry SB RAS (No. 121032500062-4).

\section{References}

1. V.N. Vodyakov, K.A. Kulikovskaya, Eng. Tech. Syst. (Russ. J.) 30, 3 (2020)

2. A.J. Peacock, Handbook of polyethylene: structure, properties, and applications (Marcel Dekker, New York, 2000)

3. A.L. Okhlopkova, O.V. Gogoleva, E.Yu. Shits, J. Frict. Wear, 25, 202 (2004) 